\title{
SMART or simply bold?
}

\author{
Valerie W. Rusch, MD, ${ }^{a}$ Andreas Rimner, MD, ${ }^{b}$ and Prasad S. Adusumilli, MD ${ }^{\mathrm{a}, \mathrm{c}}$
}

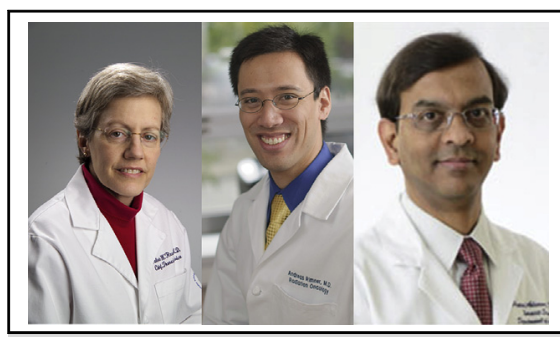

Valerie W. Rusch, MD, Andreas Rimner, MD, and Prasad S. Adusumilli, MD.

Central Message
Radiation and resection for mesothelioma
yielded good outcomes. Future studies should
validate results and elucidate immune
responses.

See Article page 468 .

See Editorials page 307 and 310. treatment, however, which is challenging to administer to a population of patients who are generally older and have multiple medical comorbidities. ${ }^{4}$

In this issue of the Journal, de Perrot and colleagues from the University of Toronto report their expanded phase II experience with the Surgery for Mesothelioma After Radiation Therapy (SMART) regimen for MPM. In a bold move, they have transposed radiotherapy to the preoperative setting, before extrapleural pneumonectomy (EPP). By using intensity-modulated radiation therapy with a high dose per radiation fraction preoperatively, they shortened the duration of treatment from approximately 6 weeks to 5 days. The rationale for this approach was to optimize the delivery of radiation to the whole tumor bed, to develop a shorter treatment plan, and to sterilize the edges of the tumor before resection. Remarkably, all 62 patients included in this study completed the planned treatment with an operative mortality of only $1.6 \%$, an overall treatment-related mortality of $4.8 \%$, and no bronchial stump leaks. The median overall survival on an intention-to-treat basis was 36 months, and the median overall survival was 51 months for patients with the prognostically favorable epithelioid histologic type.

Such outstanding results reflect the expertise of de Perrot and colleagues in the surgical care of MPM ${ }^{5}$ and the excellence of their multidisciplinary program. Implementing the SMART regimen presents several high-stakes challenges. Patient selection is critically important, because patients must be physiologically able to undergo EPP, and the surgeon must also be able to predict tumor resectability. Limitations in our current staging modalities for MPM often lead to discrepancies between clinical and pathologic staging in MPM and make it hard to predict tumor resectability

accurately. ${ }^{6}$ It is therefore notable that only 4 patients in this trial had incomplete (R2) resections. Once a patient has completed SMART-type preoperative radiotherapy, EPP is mandatory within a short time frame, because not proceeding with resection would expose the patient to lifethreatening radiation pneumonitis. To avoid bronchial stump leaks and other serious complications after EPP requires experience, along with meticulous surgical technique and postoperative care. Only high-volume centers of excellence could potentially reproduce these results.

What accounts for the very encouraging overall survival figures in this study, which are among the best reported to date for MPM? Are they solely related to patient selection, or do they reflect the true effect of a novel approach to treatment? As De Perrot and colleagues point out, the popularity of EPP has recently waned in favor of the less morbid procedure of extended pleurectomy and decortication, but their excellent results strongly suggest that there may still be a role for EPP in carefully selected patients-those with epithelioid tumor histologic type and no nodal metastases. The patterns of disease recurrence are similar to what has been reported in previous studies with EPP and standard fractionation postoperative radiation with or without chemotherapy. ${ }^{1}$ Thus the excellent overall survivals may be related to a phenomenon other than patient selection or the sequencing of treatments. MPM is known to be associated with an altered immune environment, and the patterns of immune response in tumor and related stroma are 
reported to correlate with overall survival. ${ }^{7}$ It is possible that preoperative radiation, at least in some patients, is affecting immune response which in turn is influencing overall survival. The recent dramatic success of checkpoint inhibitors including ipilimumab, nivolumab, and pembrolizumab in malignancies such as metastatic melanoma and non-small cell lung cancer emphasizes the importance of immune response in cancer treatment. It is important to determine the impact of preoperative radiation on immune response in MPM and to investigate potential synergisms with checkpoint inhibitors or other immunotherapies.

The treatment of MPM has long been surrounded by nihilism. Some would assert that the results of this study are not credible or should now be subjected to the gold standard of a randomized clinical trial. The results can be corroborated by extending the treatment approach to a few other centers with similar radiation oncology and surgical expertise. Human and animal model studies elucidating the potential interactions of preoperative radiation with immune response will likely be more productive than a randomized clinical trial of uncertain feasibility. De Perrot and colleagues may have been not only bold but SMART.

\section{References}

1. Rusch VW, Rosenzweig K, Venkatraman E, Leon L, Raben A, Harrison L, et al. A phase II trial of surgical resection and adjuvant high-dose hemithoracic radiation for malignant pleural mesothelioma. J Thorac Cardiovasc Surg. 2001;122:788-95.

2. Rice DC, Stevens CW, Correa AM, Vaporciyan AA, Tsao A, Forster KM, et al. Outcomes after extrapleural pneumonectomy and intensity-modulated radiation therapy for malignant pleural mesothelioma. Ann Thorac Surg. 2007;84: 1684-92; discussion 1692-3.

3. Rimner A, Spratt DE, Zauderer MG, Rosenzweig KE, Wu AJ, Foster A, et al. Failure patterns after hemithoracic pleural intensity modulated radiation therapy for malignant pleural mesothelioma. Int J Radiat Oncol Biol Phys. 2014; 90:394-401.

4. Krug LM, Pass HI, Rusch VW, Kindler HL, Sugarbaker DJ, Rosenzweig K, et al Multicenter phase II trial of neoadjuvant pemetrexed plus cisplatin followed by extrapleural pneumonectomy and radiation for malignant pleural mesothelioma. $J$ Clin Oncol. 2009;27:3007-13.

5. de Perrot M, Uy K, Anraku M, Tsao MS, Darling G, Waddell TK, et al Impact of lymph node metastasis on outcome after extrapleural pneumonectomy for malignant pleural mesothelioma. J Thorac Cardiovasc Surg. 2007; 133:111-6.

6. Rusch VW, Giroux D, Kennedy C, Ruffini E, Cangir AK, Rice D, et al; IASLC Staging Committee. Initial analysis of the International Association for the Study of Lung Cancer Mesothelioma Database. J Thorac Oncol. 2012;7:1631-9.

7. Ujiie H, Kadota K, Nitadori JI, Aerts JG, Woo KM, Sima CS, et al. The tumoral and stromal immune microenvironment in malignant pleural mesothelioma: a comprehensive analysis reveals prognostic immune markers. Oncoimmunology. 2015;4:e1009285. 